\title{
Modes of longitudinal combined discharge in low pressure nitrogen
}

\author{
V A Lisovskiy, N D Kharchenko and V D Yegorenkov
}

Kharkov National University, 4 Svobody sq., Kharkov, 61077, Ukraine

Received 28 December 2007, in final form 3 April 2008

Published 29 May 2008

Online at stacks.iop.org/JPhysD/41/125207

\begin{abstract}
This paper reports the modes of a low pressure discharge in the combined $(\mathrm{rf}+\mathrm{dc}$ ) electric field. We propose to distinguish three modes of a longitudinal combined discharge ( $\mathrm{rf}$ and dc voltages were applied to the same electrodes): (1) a non-self-sustained rf discharge perturbed by a dc electric field, (2) a combined discharge and (3) a non-self-sustained dc discharge perturbed by an rf electric field. The existence conditions of these modes are determined. The parameter range in which the first mode of the combined discharge may be extinguished via increasing dc voltage is shown to be limited with an rf discharge extinction curve from the low pressure side as well as with a curve of the least rf voltage corresponding to the transition of the combined discharge from the first mode to the second one. The relation between the thicknesses of the 'cathode' and 'anode' near-electrode sheaths is derived analytically for the first mode, which is in good agreement with experimental data.
\end{abstract}

\section{Introduction}

The gas discharge in the combined $(\mathrm{rf}+\mathrm{dc})$ electric field is applied for determining the electron transport coefficients in low pressure gases [1,2], as well as in a number of technological devices for spectral-chemical analysis [3], silicon plasma oxidation [4], plasmatrons [5] and gas discharge lasers $[6,7]$. These applications led to the emergence of a large number of experimental and theoretical papers devoted to studying the characteristics of such combined discharges in various gases (see, e.g. [8-18]). The combination of $\mathrm{dc}$ and $\mathrm{rf}$ electric fields increases the stability of gas discharge burning permitting one to introduce a large power into the discharge $[6,7,10,11]$. The papers [8,9] studied the transverse combined discharge in which the inner dc electrodes were located at the ends of the discharge tube and the rf electrodes were located outside the tube. Thus, crossed rf and dc electric fields were created in the discharge tube. The authors of $[8,9]$ presented the radial profiles of the integral glow of the transverse combined discharge. The papers $[12,14]$ are devoted to the experimental and theoretical study of the ignition of the longitudinal combined discharge in argon and air. The breakdown curves of the combined longitudinal discharge in argon are presented in [17] for purely metallic electrodes as well as for electrodes coated with a dielectric layer. Employing numerical modelling, the authors of paper [18] made an attempt to describe the experimental breakdown curves [12]. The author of paper [13] recorded with a Langmuir probe the axial profiles of plasma concentration of the longitudinal combined discharge in air; the measurements were performed with the gas pressure close to and to the right of the extinction curve minimum for the rf capacitive discharge. The paper [15] studies the discharge characteristics in the combined $(\mathrm{rf}+\mathrm{dc})$ electric field of a complicated configuration (triode scheme). Here the distribution functions over energy were recorded for ions incident on the grounded electrode at different values of rf and dc voltages.

Though the structure and modes of the rf capacitive discharge [19] and dc glow discharge [20] are studied quite well, still the question on the modes of the longitudinal combined discharge when $\mathrm{rf}$ and dc voltages are applied to the same electrodes remains open.

In a combined discharge the rf electric field plays the main role in producing a dense plasma whereas the dc voltage across the electrodes increases the energy of positive ions incident on the 'cathode'. The dc voltage 'drops' mainly across the 'cathode' sheath (due to its low conductivity), whereas a considerable portion of the rf voltage applied 'drops' across the quasi-neutral plasma controlling the ionization rate of gas molecules via electron impact. In contrast to the rf capacitive discharge and the dc discharge, it is possible to independently control in the combined discharge the ion energy (with the dc voltage $U_{\mathrm{dc}}$ ) and the ion flow onto the electrode (controlling the plasma concentration with rf voltage $U_{\mathrm{rf}}$ ). 


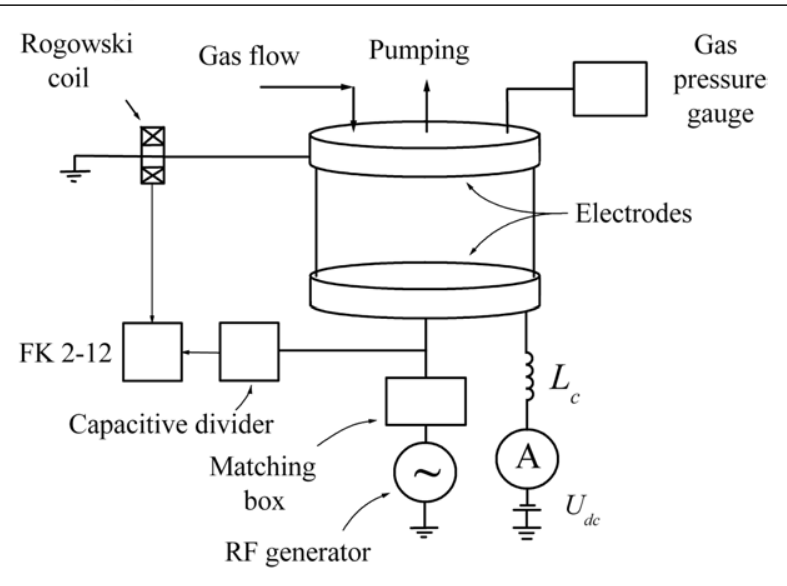

Figure 1. Scheme of the experimental device.

Low pressure discharges in nitrogen are widely applied in industrial processes for the production of hard nitride layers on steel, deposition of thin silicon nitride $\mathrm{Si}_{3} \mathrm{~N}_{4}$ films, amorphous carbon nitride and $\beta-\mathrm{C}_{3} \mathrm{~N}_{4}$ III-nitrides. Therefore, we chose nitrogen for studying the burning modes of the combined discharge.

Our paper aims to study the modes of the longitudinal combined discharge in low pressure nitrogen. It is shown that the combined discharge may exist in one of the three possible modes at different values of the ratio of the rf voltage to the dc voltage. The first mode-the rf discharge perturbed with a dc electric field - is observed at moderate dc voltages. The second mode - the combined discharge-exists in the presence of intense ionization in the 'cathode' sheath, when the dc voltage exceeds a certain critical value. The third modethe dc discharge perturbed with rf voltage-is observed when a moderate rf voltage is applied to the burning dc discharge. It is shown that the range exists limited from the low pressure side where the first mode may be extinguished via increasing dc voltage. A relation is derived from the collisional ChildLangmuir law between the thicknesses of the 'cathode' and the 'anode' sheaths furnishing a good description of the experimental data for the first mode.

\section{Experimental conditions}

The experiments were performed at nitrogen pressure within the range $p=0.01-5$ Torr with amplitude values of the $\mathrm{rf}$ voltage $U_{\text {rf }} \leqslant 2000 \mathrm{~V}$, the dc voltage $U_{\mathrm{dc}} \leqslant 600 \mathrm{~V}$ and the rf field frequency $f=13.56 \mathrm{MHz}$. The distance between flat parallel stainless-steel electrodes was $L=30 \mathrm{~mm}$. The rf potential was applied to one of the electrodes whereas the other one was grounded. The rf electrode served as a 'cathode' simultaneously, because a negative dc potential was applied to it.

Figure 1 shows our experimental device. The fused silica tube with an inner diameter of $100 \mathrm{~mm}$ was vacuum-sealed between the electrodes. The gas supply system fed nitrogen through a multitude of tiny orifices in the grounded electrode. The discharge vessel was evacuated through a set of orifices in the same electrode. This design permitted us to feed and pump out the gas uniformly across the electrode area, which is important in technological processes. For registering the gas pressure within the range from $10^{-3}$ Torr to the atmospheric one we employed the thermoelectric vacuum gauge. Gas was pumped out with the preliminary vacuum and turbo molecular pumps providing a limiting pressure order of $10^{-6}$ Torr. An rf generator was connected to the potential electrode via a matching box of the $\Pi$-type. The dc source was connected to the same electrode via a choke of $L_{\mathrm{c}}=4 \mathrm{mH}$, to prevent damage to the source by the rf current.

The electron temperature $T_{\mathrm{e}}$, the plasma potential and the plasma concentration $n_{\mathrm{i}}$ were established from the measurements with a single cylindrical nichrome Langmuir probe (the probe length was $5.5 \mathrm{~mm}$, the probe diameter was $0.18 \mathrm{~mm}$ ). The plasma concentration $n_{\mathrm{i}}$ was calculated from the ion branch of the probe current $I_{\mathrm{pr}}$ and the electron temperature $T_{\mathrm{e}}$ determined according to the technique described in the papers [21,22]. To this end we employed the formula

$$
I_{\mathrm{i}, \text { measured }}=I_{\mathrm{i}}^{*} \cdot I^{*},
$$

where

$$
\begin{array}{r}
I^{*}=A \sqrt{\frac{k T_{\mathrm{e}}}{2 \pi M_{\mathrm{i}}}} n_{\mathrm{i}} e, \\
I_{\mathrm{i}}^{*}=\gamma_{1} \gamma_{2} I_{\mathrm{L}}^{*},
\end{array}
$$

where $A$ denotes the probe collecting surface, $k$ is the Boltzmann constant, $M_{\mathrm{i}}$ is the ion mass, $e$ is the elementary charge, the coefficients $\gamma_{1}$ and $\gamma_{2}$ are functions of the ion concentration, electron temperature and gas pressure [21]:

$$
\gamma_{1}, \gamma_{2}=f_{1,2}\left(n_{\mathrm{i}}, T_{\mathrm{e}}, p\right)
$$

$I_{\mathrm{L}}^{*}$ is the Laframboise current

$$
I_{\mathrm{L}}^{*}=\frac{2}{\sqrt{\pi}} \sqrt{\eta}
$$

and $\eta=e U_{\mathrm{p}} / k T_{\mathrm{e}}$ is the dimensionless probe current equalling zero at the plasma potential. Inserting (2)-(4) into (1) furnishes the equation the left-hand part of which contains the ion current that we registered with the probe and the right-hand part of which is the function of the gas pressure, electron temperature and plasma concentration. Solving this equation we get the concentration of positive ions. In doing so we use the values of the electron temperature determined from the probe CVCs (employing the linear section of the graph of the electron current to the probe constructed to the semi-logarithmic scale)

\section{Experimental results}

We aim to study the optional modes of a longitudinal combined discharge and the existence conditions of each mode, to register the discharge current-voltage characteristics (CVCs) (with respect to dc as well as rf currents) as well as the thickness of the 'cathode' and the 'anode' sheaths under various conditions. We start with the case when only the self-sustained RF discharge is burning. 


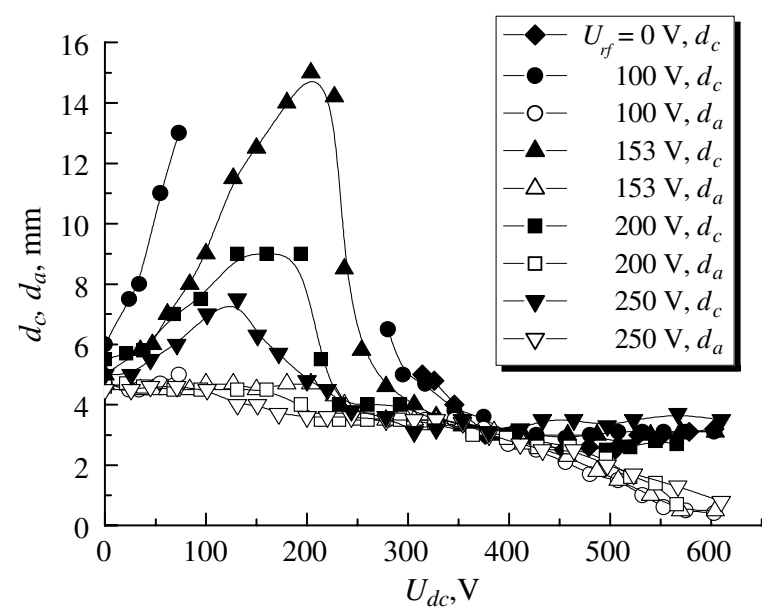

Figure 2. 'Cathode' $d_{\mathrm{c}}$ and 'anode' $d_{\mathrm{a}}$ sheath thicknesses against the applied dc voltage with fixed values of rf voltage and $\mathrm{N}_{2}$ pressure of 0.5 Torr. Solid points are for $d_{\mathrm{c}}$ and empty points are for $d_{\mathrm{a}}$.

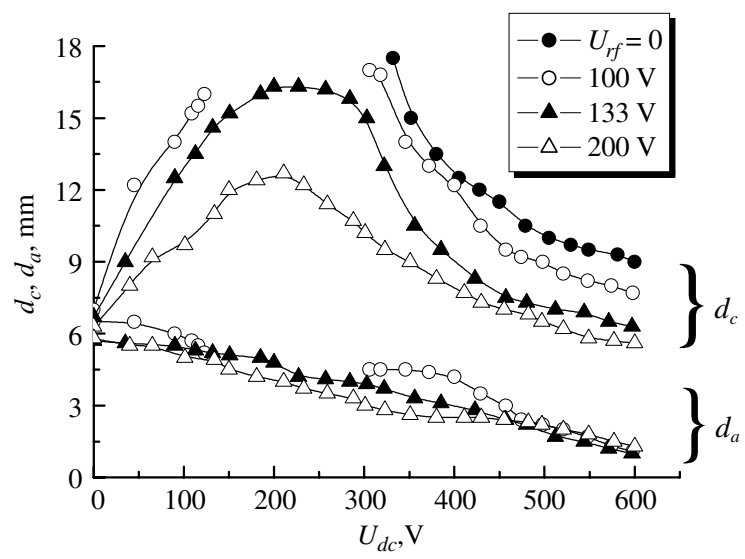

Figure 3. 'Cathode' $d_{\mathrm{c}}$ and 'anode' $d_{\mathrm{a}}$ sheath thickness against applied dc voltage with fixed values of rf voltage and $\mathrm{N}_{2}$ pressure of 0.1 Torr.

The rf capacitive discharge possesses a layered structure and it consists of near-electrode sheaths of almost equal thickness and a region of a quasi-neutral plasma. Let us ignite the rf discharge in the weak-current $\alpha$-mode, in which the ionization occurs in the region of the quasi-neutral plasma and the conductivity of near-electrode sheaths is small [19,20,23]. Now let us apply a small dc voltage across the electrodes. As a result the thickness of the sheath near the 'cathode' $d_{\mathrm{c}}$ increases, and the thickness of the sheath near the 'anode' $d_{\mathrm{a}}$ remains almost unchanged or decreases weakly (see figures 2 and 3). In this case we observe the first mode of the longitudinal combined discharge-the $r f$ discharge perturbed with the $d c$ electric field. If the rf voltage does not exceed the limiting value $U_{\text {rf.min }}$, the further increase in the dc voltage decreases the plasma region width, and at some $U_{\mathrm{dc}}$ value the discharge goes out. The less is the rf voltage sustained, the less dc voltage $U_{\mathrm{dc}}$ is required for discharge extinguishing. However, with further growth in $U_{\mathrm{dc}}$ the joint action of dc and rf voltages entails the gas breakdown, and the discharge is ignited in the second mode, which we tentatively call 'the combined discharge'.

Figure 4 shows the extinction curve $U_{\text {rf.ext }}(p)$ of a discharge. The rf discharge may exist only at rf voltage

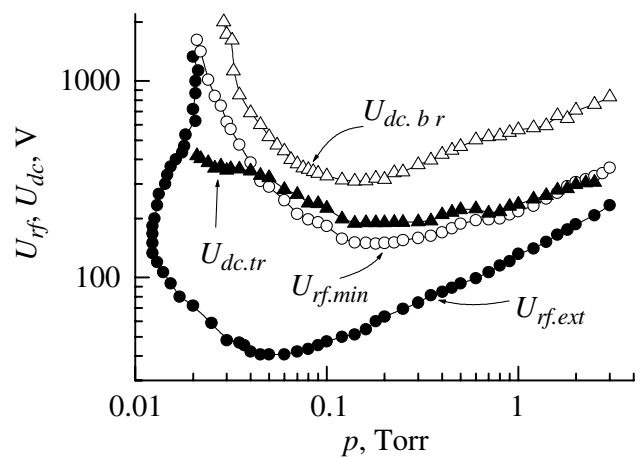

Figure 4. Rf extinction voltage $U_{\text {rf.ext }}$ of the self-sustained $\mathrm{rf}$ discharge, the minimum rf voltage $U_{\text {rf.min }}$ for the transition from the first mode of the combined discharge to the second one, the $\mathrm{dc}$ voltage $U_{\text {dc.tr }}$, corresponding to this transition and the dc voltage igniting the self-sustained dc discharge $U_{\text {dc.br }}$ against gas pressure.

values exceeding $U_{\text {rf.ext }}$. At a low gas pressure (to the left of the minimum) the extinction curve possesses a region of multi-valued dependence of the extinction voltage on the gas pressure $[23,24]$. The reasons for such a behaviour of the extinction are given clearly in [24]; therefore we will not pay attention to it here. Figure 4 shows the critical rf voltage $U_{\text {rf.min }}$ against nitrogen pressure. At $U_{\text {rf }}=U_{\text {rf.min }}$ the discharge does not go out with the growth in dc voltage but experiences a transition to the second mode. The dc voltage value required for the transition from the first mode to the second one equals $U_{\mathrm{dc}}=U_{\mathrm{dc} . t r}$ and it is shown in figure 4. It is clear from the figure that at a low nitrogen pressure and a high rf voltage the rf discharge extinction curve $U_{\text {rf.ext }}$ and the critical rf voltage curve of the transition to the second mode $U_{\text {rf.min }}$ merge. Therefore, the region of pressure and the rf voltage in which the first mode of the combined discharge can be extinguished by application of the dc voltage is limited from the low pressure side and it is contained between the $U_{\text {rf.ext }}$ and $U_{\text {rf.min }}$ curves. It does not mean that the existence region of the second mode of the combined discharge is located only in the gap between the $U_{\text {rf.ext }}$ and $U_{\text {rf.min }}$ curves. The first mode is also observed at the rf voltage values $U_{\text {rf }}>U_{\text {rf.min }}$, but with the dc voltage increasing the discharge experiences a transition to the second mode. Besides, whereas in the first mode the 'cathode' sheath is dark and its width grows with increasing $U_{\mathrm{dc}}$, the glow of violet tint appears in the 'cathode' sheath after the transition to the second mode (thus indicating the appearance of high energy electrons in the sheath), and its thickness decreases with the dc voltage increasing. Figures 2 and 3 clearly demonstrate the weak variation of the 'anode' sheath thickness in the first mode in contrast to its decrease with the dc voltage increasing in the second mode.

Figure 4 also shows the dc voltage required for the transition from the first mode to the second one (during which the breakdown of the 'cathode' sheath occurs judging from the variation in the discharge colour), which happens to be much less than the ignition voltage of the self-sustained dc discharge. For instance, at the nitrogen pressure of 0.14 Torr (near the minimum of the dc breakdown curve) the breakdown voltage value equals $U_{\mathrm{dc} . b r}=310 \mathrm{~V}$, and the voltage value corresponding to the discharge transition to the second mode 


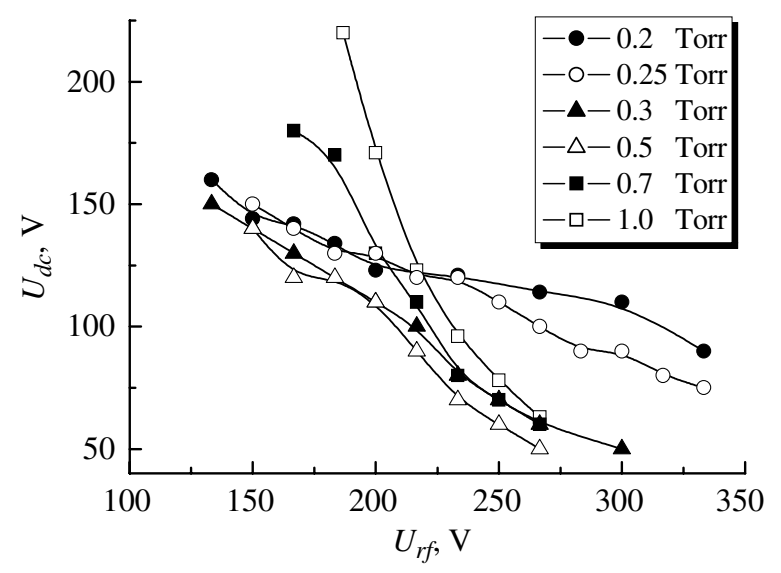

Figure 5. Dc voltages for the transition from the first mode of the combined $\mathrm{rf} / \mathrm{dc}$ discharge to the second one against the applied rf voltage at various nitrogen pressure values.

equals $U_{\text {dc.tr }}=189 \mathrm{~V}$. First, it is due to the circumstances that not only is the dc voltage drop concentrated across the 'cathode' sheath, but at certain time moments almost all of the rf voltage applied to the electrodes drops across it. Second, the flow of positive ions and high energy photons enters the 'cathode' sheath from the plasma region (which knock out secondary electrons hitting the electrode surface), as well as metastable atoms and molecules (which enhance the flow of secondary electrons under deactivation as well as via set ionization in the 'cathode' sheath). As known [25], metastable electron states $\mathrm{N}_{2}\left(A^{3} \Sigma_{\mathrm{u}}^{+}\right)$and $\mathrm{N}_{2}\left(a^{\prime 1} \Sigma_{\mathrm{u}}^{-}\right)$play an important role in sustaining the dc discharge; meanwhile ionization processes (including associative ionization) occur according to the reactions

$$
\begin{aligned}
\mathrm{N}_{2}\left(A^{3} \Sigma_{\mathrm{u}}^{+}\right)+\mathrm{N}_{2}\left(a^{\prime}{ }^{1} \Sigma_{\mathrm{u}}^{-}\right) & \rightarrow \mathrm{N}_{2}^{+}+\mathrm{N}_{2}+\mathrm{e} \\
& \rightarrow \mathrm{N}_{4}^{+}+\mathrm{e}
\end{aligned}
$$

and

$$
\begin{aligned}
\mathrm{N}_{2}\left(a^{\prime 1} \Sigma_{\mathrm{u}}^{-}\right)+\mathrm{N}_{2}\left(a^{\prime 1} \Sigma_{\mathrm{u}}^{-}\right) & \rightarrow \mathrm{N}_{2}^{+}+\mathrm{N}_{2}+\mathrm{e} \\
& \rightarrow \mathrm{N}_{4}^{+}+\mathrm{e} .
\end{aligned}
$$

These reactions may take place in the plasma volume as well as in the cathode sheath of the combined discharge, making the transition from the first mode to the second one easier. The amount of energy freed under quenching metastables $\mathrm{N}_{2}\left(a^{\prime}{ }^{1} \Sigma_{\mathrm{u}}^{-}\right)$and $\mathrm{N}_{2}\left(A^{3} \Sigma_{\mathrm{u}}^{+}\right)$on the 'cathode' surface ( $8.4 \mathrm{eV}$ and $6.2 \mathrm{eV}$ [20], respectively) is sufficient to produce secondary electrons. Therefore, it is easier to break the 'cathode' sheath of the combined discharge in the first mode than to ignite the dc discharge.

Figure 5 exposes the dc voltage values at which the transition from the first mode of the combined discharge to the second one occurs against the applied rf voltage at various values of nitrogen pressure. The figure shows that the higher is the rf voltage, the lower is the dc voltage to be applied to produce the discharge transition to the second mode. At low rf voltage values the plasma concentration is small, and the application of the dc voltage induces fast growth in the 'cathode' sheath thickness. In order to break a thick 'cathode' sheath, a higher dc voltage is required, which we observe in the figure.

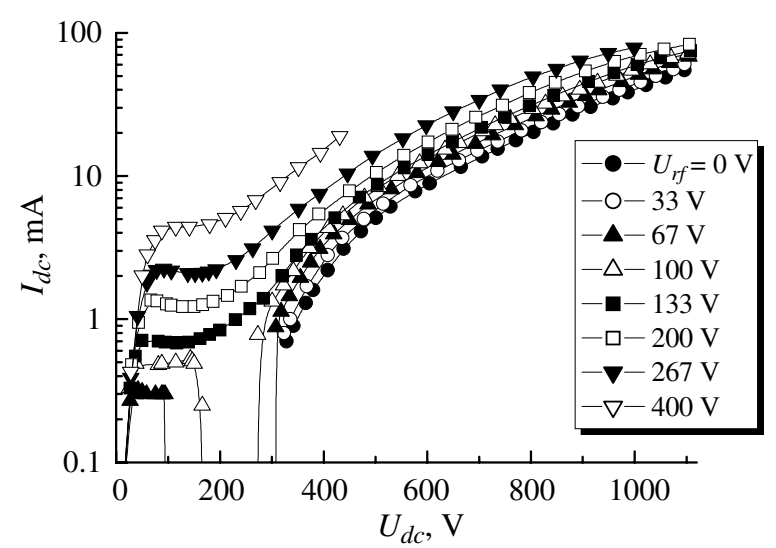

Figure 6. CVCs of the combined discharges for nitrogen pressure $p=0.1$ Torr.

Now consider the CVCs of the combined discharge. Figure 6 shows the direct current $I_{\mathrm{dc}}$ against the direct voltage $U_{\mathrm{dc}}$ at the nitrogen pressure of 0.1 Torr. At low rf voltage values (below $50 \mathrm{~V}$ ) it is impossible to support the self-sustained discharge; therefore, we observe in the figure the CVCs for the self-sustained dc discharge (at $U_{\text {rf }}=0 \mathrm{~V}$ ) and for the third mode of the longitudinal combined discharge-non-selfsustained dc discharge perturbed with rf voltage. On applying the rf voltage to the dc discharge it is possible to sustain the discharge burning at a smaller dc voltage than for a selfsustained dc discharge (therefore, we call the third mode the 'non-self-sustained dc discharge'). At higher rf voltages (in the figure it corresponds to the curves for $U_{\mathrm{rf}} \geqslant 67 \mathrm{~V}$ ) it is possible to support a self-sustained rf discharge. First, increasing the dc voltage $U_{\mathrm{dc}}$ involves a small growth in the dc current through the discharge, then the current achieves a maximum and with further growth in $U_{\mathrm{dc}}$ the current decreases. This current decrease is associated with the increase in the 'cathode' sheath thickness and the decrease in the plasma region width. The number of charged particles within the plasma volume decreases thus leading to the reduction in the discharge current. Then at some dc voltage value the discharge goes out, but at higher $U_{\mathrm{dc}}$ voltages it ignites again but in the second mode, and the dc voltage increase is accompanied by the increase in discharge current.

At the rf voltage above $133 \mathrm{~V}$ the first mode of the combined discharge does not go out, and at a sufficiently large dc voltage a breakdown of the 'cathode' sheath occurs (electron avalanches develop in the total voltage drop in $\mathrm{rf}$ and $\mathrm{dc}$ voltages across the 'cathode' sheath). This breakdown at low pressure is accomplished without a jump; the discharge current increases and the discharge experiences a smooth transition from the first mode to the second one.

Figure 7 demonstrates the CVCs of the longitudinal combined discharge at the nitrogen pressure of $p=1$ Torr. In the absence of the rf voltage the self-sustained dc discharge before extinguishing burns in the normal mode, occupying only a part of the electrode area. Besides, the current increases due to the expansion of the current spot area on the electrodes with an almost unchanged value of the dc voltage (actually a small decrease in the dc voltage across the electrodes is 


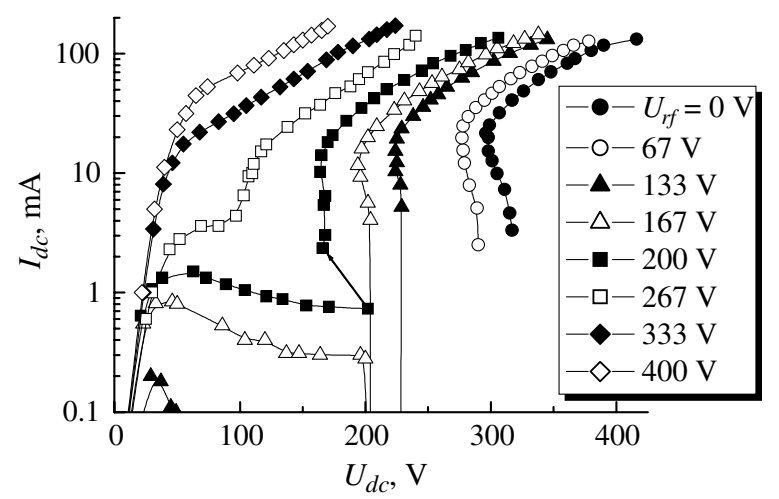

Figure 7. CVCs of the combined discharges for nitrogen pressure $p=1$ Torr.

observed due to the increase in the discharge gap conductance when the discharge expands over the electrode area). When the discharge occupies the total area of the electrodes, the further growth in the current is accompanied by the increase in the dc voltage. The figure shows that the application of a moderate rf voltage (in the figure it corresponds to the case with $U_{\mathrm{rf}}=67 \mathrm{~V}$ ) entails not only the growth in discharge current but also the decrease in the minimum dc voltage for discharge burning (in this case the normal voltage). Under these conditions we again observe the third mode-the nonself-sustained dc discharge perturbed with the rf electric field. At higher rf voltages the discharge experiences a transition from the third mode to the second one.

Starting from the rf voltage $U_{\text {rf }}=133 \mathrm{~V}$, the self-sustained rf discharge may burn, which under application of the dc voltage $U_{\mathrm{dc}}$ across it transforms to the first mode of the combined discharge. At the same time, with increasing $U_{\mathrm{dc}}$ the dc current first increases, but starting with $U_{\mathrm{dc}} \approx 50 \mathrm{~V}$, it decreases uniformly down to the discharge extinguishing. On increasing the dc voltage further the discharge ignites again but in the second mode. At the rf voltage $U_{\mathrm{rf}}=167 \mathrm{~V}$ the first mode behaves similarly, but it goes out at a higher dc voltage $(197 \mathrm{~V})$, and then it is sufficient to increase the dc voltage by only $5 \mathrm{~V}$ to break down the gas and ignite the second mode characterized by a fast growth in the dc current. At higher rf voltages the transition of the combined discharge from the first mode to the second one occurred without preceding extinguishing of the first mode and it was accompanied by the breakdown of the 'cathode' sheath. The thickness of the 'cathode' sheath decreased with a jump and the discharge current increased. With increasing rf voltage this jump-like transition of the first mode to the second one becomes smoother.

Now let us consider the transition of the combined discharge from the third mode to the second one in more detail. We are interested in how the rf voltage affects the characteristics of the burning dc discharge. Figure 8 demonstrates the dependence of the dc current on the rf voltage applied at various fixed values of the dc voltage. It is clear from this figure that the application of a moderate rf voltage gives rise to a weak growth in the dc current, the structure of the discharge glow experiencing no remarkable external changes (the discharge consists of a comparatively dark cathode sheath,

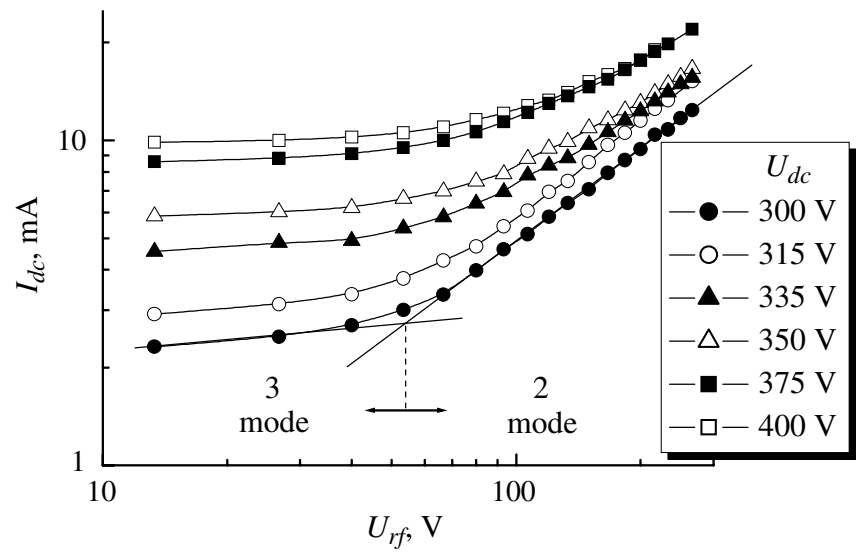

Figure 8. Dc current against the rf voltage at a nitrogen pressure of 0.2 Torr and various dc voltage values.

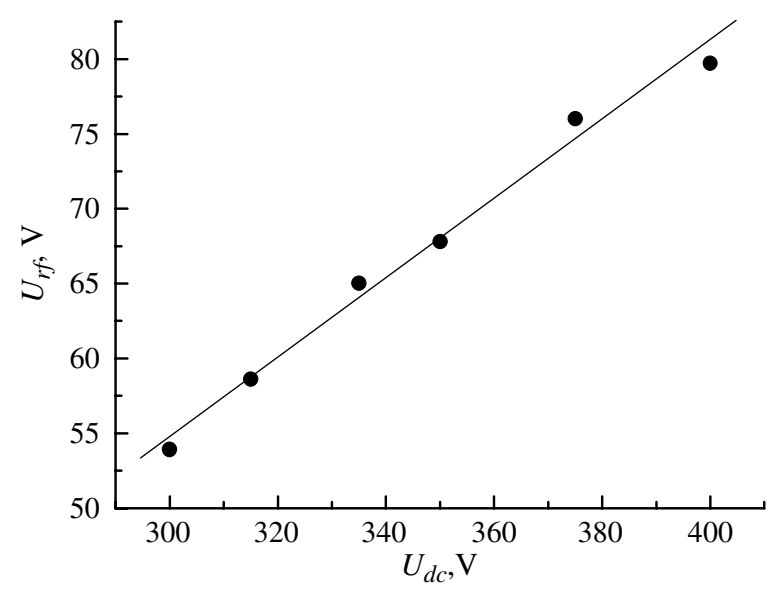

Figure 9. Rf voltage for the combined discharge transition from the third mode to the second one against dc voltage at a nitrogen pressure of 0.2 Torr.

negative glow and a dark Faraday space extending almost up to the anode itself near the surface of which the anode glow is observed). But a subsequent increase in the rf voltage entails fast growth in the dc current, and a glow appears near the anode at a distance close to the near-electrode sheath thickness of the self-sustained rf discharge (it may be proved by switching off the dc voltage generator and observing that the rf discharge in the chamber continues to burn). The curves in figure 8 constructed in a logarithmic scale consist of two linear sections, and the transition of the combined discharge from the third mode to the second one takes place at the intersection of them. The rf voltage producing this transition experiences an approximately linear growth with the dc voltage (within the $U_{\mathrm{dc}}$ range we studied), which is shown in figure 9.

We also registered the plasma inner parameters with a Langmuir probe. The axial profiles of the plasma concentration, the electron temperature and the dc plasma potential are presented in figure 10. The grounded anode is located on the left $(z=0)$, and the cathode location is marked with the coordinate $z=30 \mathrm{~mm}$. The curves with $U_{\mathrm{dc}}=0$ are the axial profiles for the self-sustained rf discharge with the distribution of the plasma concentration and the dc plasma potential that is symmetric with respect to the discharge centre, 


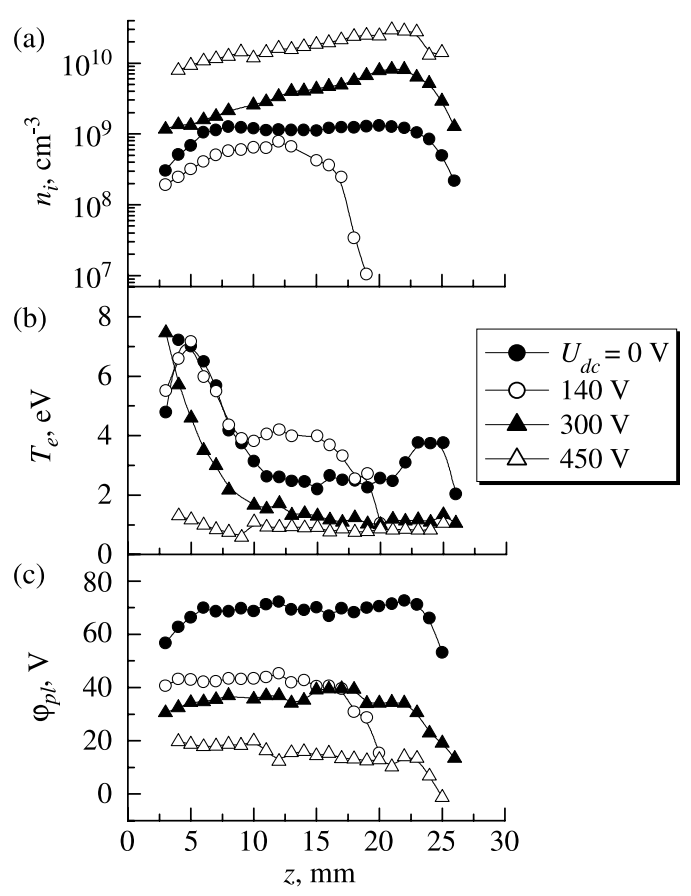

Figure 10. Axial profiles of the plasma concentration $(a)$, electron temperature $(b)$ and dc plasma potential $(c)$ at a nitrogen pressure of 0.2 Torr, rf voltage $U_{\mathrm{rf}}=133 \mathrm{~V}$ and various dc voltage values.

whereas the peaks of electron temperature are located in the vicinity of near-electrode sheath boundaries. As shown in [26], the intense heating of electrons near the sheath boundaries is associated with the fact that in the $\alpha$-mode the cold electrons acquire their energy when the near-electrode sheath expanding in the cathode phase sweeps them out into the plasma volume. The curves for $U_{\mathrm{dc}}=140 \mathrm{~V}$ are related to the first mode of the combined discharge, the cathode sheath thickness actually comprising one-half of the inter-electrode gap width. The application of the dc voltage led to the decrease in the plasma concentration not only in the extended cathode sheath but also in the plasma. This gave rise to the increase in the electron temperature in the plasma volume because the plasma with a lowered concentration is less effective in expelling from it the rf electric field heating the electrons. However, the peak of the electron temperature near the anode sheath boundary persisted indicating the weak influence of the dc voltage on the processes in the anode sheath in the first mode of the combined discharge. The curves for $U_{\mathrm{dc}}=300 \mathrm{~V}$ describe the second mode of the combined discharge. The breakdown of the cathode sheath and the development of electron avalanches in it involved the restructuring of the discharge. A peak of the plasma concentration appeared near the boundary of the compressed cathode sheath, the electron temperature decreased abruptly in this region (similar to the characteristics of the dc discharge) and the dc plasma potential also decreased. The rf electric field contributes to electron heating remarkably only in the plasma region close to and within the anode sheath giving rise to the electron temperature on approaching the anode. The anode glow is located close to the anode making it difficult to study with a probe because of a considerable violation of plasma quasi-neutrality in it and the anisotropic distribution of charged particles over velocity. However, we know that the anode in the dc discharge serves as a collector of electrons equalling the electron current in the external circuit and the total discharge current. A large portion of fast electrons having left the cathode sheath just cannot approach this part of the discharge because of energy loss due to inelastic and elastic collisions with gas molecules and thermalization. In this case, there is a necessity for additional ionization in the anode sheath to make equal the electron current in the external circuit and the total discharge current, and an anode glow may then be observed near the anode surface. A further growth in the dc voltage $\left(U_{\mathrm{dc}}=450 \mathrm{~V}\right)$ leads to the increase in the plasma concentration within the gap (with a peak in the negative glow, near the boundary of the cathode sheath), the electron temperature becomes lower than $1 \mathrm{eV}$ (except for a small area near the anode sheath) and the dc plasma potential with respect to the anode also reduces. A large diffusion electron current flows to the anode (out of the region with an enhanced plasma concentration), together with the flow of fast electrons hit out of the cathode surface and accelerated within the cathode sheath. No considerable anode voltage drops are required to convey these two electron flows to the anode surface, and the anode glow actually disappears.

Here is the summary of the main properties of the three modes of the combined discharge.

(1) Non-self-sustained $r f$ discharge perturbed by the $d c$ electric field. The cathode sheath is not broken; there is no ionization in it. The main ionization occurs within the plasma volume due to electrons having gained their energy in the effective rf electric field as well as due to stochastic heating of electrons in the cathode phase of sheaths. Increasing dc voltage leads to the increase in the thickness of the cathode sheath, the enhanced loss of positive ions from the plasma and, as a consequence, the decrease in the discharge current. This mode of the combined discharge may be extinguished within a certain range of rf voltages $\left(U_{\mathrm{rf}}<U_{\mathrm{rf} \text {.min }}\right)$ by the increase in the dc voltage, which limits its application in plasma technologies.

(2) Combined discharge. The cathode sheath is broken; electron avalanches develop in it contributing to the ionization of gas molecules substantially. The remaining sufficiently large rf electric field supports the ionization in the plasma volume. The cathode sheath thickness decreases with the dc voltage growth due to the fast growth in the plasma concentration in the volume; the discharge current also grows.

(3) Non-self-sustained $d c$ discharge perturbed by the $r f$ electric field. The ionization in the cathode sheath in the dc electric field plays the main role in sustaining the combined discharge. The weak rf field makes a certain contribution to the gas ionization in the discharge volume. The cathode sheath thickness decreases slightly with increasing rf voltage, and the discharge current increases.

\section{Consideration of the Child-Langmuir equation}

Now let us take the Child-Langmuir equation for the collisional rf sheath [19] (equation (3.17)) in order to interpret 


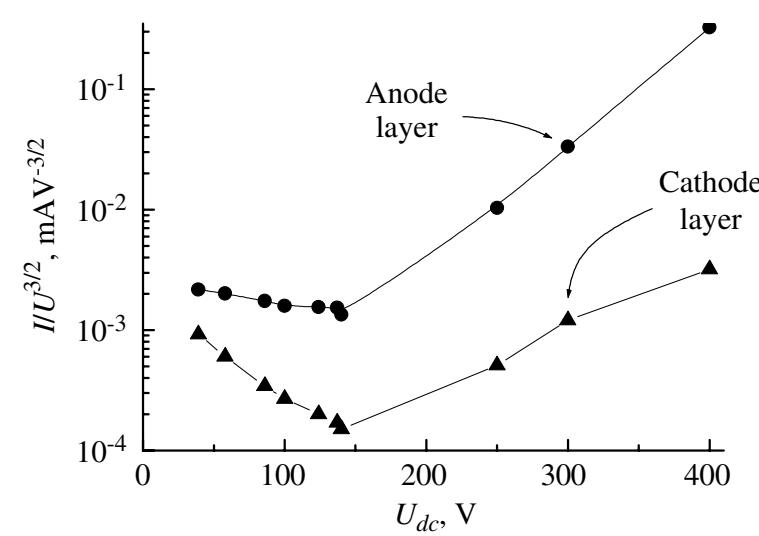

Figure 11. Ratio of dc current to dc voltage in power of $3 / 2$ against the dc voltage across the sheath for the cathode and anode sheaths at a nitrogen pressure of 0.2 Torr.

a part of the presented results:

$$
J=\frac{1}{4 \pi}\left(\frac{500}{243 \pi}\right)^{1 / 2}\left(\frac{2 e l}{M}\right)^{1 / 2} \frac{\bar{V}^{3 / 2}}{d^{5 / 2}},
$$

where $J$ is the ion current density through the sheath, $e$ is the elementary charge, $l$ is the mean free path of positive ions in the sheath, $M$ is the ion mass, $\bar{V}$ is the dc voltage drop across the near-electrode sheath and $d$ is the sheath thickness. We assume the plasma volume to possess high conductance and the rf voltage drop across the plasma to be much less than that across the sheath.

Let us first employ the Child-Langmuir equation (5) to clarify the behaviour of the cathode and anode sheath thicknesses in the first and second modes of the combined discharge. Let us ignite the dc discharge at the pressure value when it covers the surface of the electrodes completely (say, 0.2 Torr) and the rf voltage value $U_{\text {rf }}>U_{\text {rf.min }}$ and measure the dc current $I_{\mathrm{dc}}$ in the electrode circuit with increasing dc voltage. Then the value of the dc current $I_{\mathrm{dc}}$ is related to the current density $J$ from formula (5) as $I_{\mathrm{dc}}=J S$, where $S$ is the electrode area. Let us determine the dc voltage drop $\bar{V}$ across the cathode and anode sheaths using the probe measurements (figure 10). From (5) we obtain a simple formula $I_{\mathrm{dc}} / \bar{V}^{3 / 2} \propto$ $d^{-5 / 2}$. If the quantity $I_{\mathrm{dc}} / \bar{V}^{3 / 2}$ decreases with increasing dc voltage then the thickness of the corresponding sheath increases and vice versa. Figure 11 shows the $I_{\mathrm{dc}} / \bar{V}^{3 / 2}$ values for the cathode and anode sheaths. In the range of the dc voltage $U_{\mathrm{dc}} \leqslant 140 \mathrm{~V}$ the first mode of the combined discharge is observed, and the second one at higher $U_{\mathrm{dc}}$ values. It is clear from figure 11 that in the first mode the quantity $I_{\mathrm{dc}} / \bar{V}^{3 / 2}$ decreases fast for the cathode sheath and experiences weak changes for the anode sheath. It explains the increase in the cathode sheath thickness $d_{\mathrm{c}}$ and the almost unchanged value of the anode sheath thickness $d_{\mathrm{a}}$ with $U_{\mathrm{dc}}$ increasing in the first mode in figures 2 and 3 . In the second mode the thickness values of the cathode as well as anode sheaths decrease which follows from the growth in $I_{\mathrm{dc}} / \bar{V}^{3 / 2}$ in figure 11 .

In a self-sustained symmetric rf discharge the thicknesses of near-electrode sheaths are equal, and the dc voltage drop across the near-electrode sheath is $\bar{V}=U_{\text {rf }} / k$, where $k$ is a

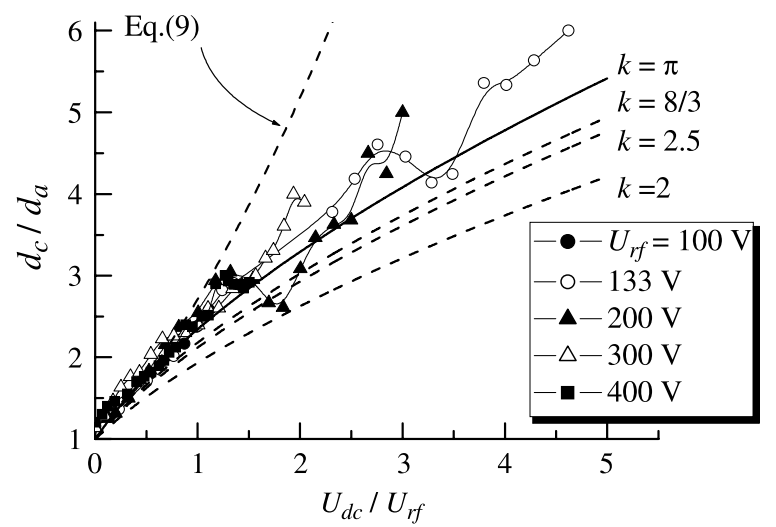

Figure 12. 'Cathode' sheath to 'anode' sheath thickness ratio against the $U_{\mathrm{dc}} / U_{\mathrm{rf}}$ ratio for various rf voltage values and nitrogen pressure of 0.1 Torr. We also present the results of calculations according to formula (8) (for different $k$ values) and formula (9) (obtained from the data of paper [31]).

certain constant. There is no agreed value among the authors regarding this constant. For instance, $k=\pi$ is assumed in [27], $k=8 / 3$ in $[19,28]$, the consideration of the electric circuit of the rf discharge furnishes the value $k=2[19,29]$ and the model of the collisional sheath gives $k=2.5$ [30]. In the first mode of the combined discharge the 'anode' sheath thickness $d_{\mathrm{a}}$ remains actually unchanged, i.e. the application of the dc voltage essentially affected only the characteristics of the 'cathode' sheath. Consequently, only the rf voltage whose dc component is $\overline{V_{\mathrm{a}}}=U_{\mathrm{rf}} / k$ drops across the 'anode' sheath. In the first mode of the combined discharge the dc applied voltage and the dc component of the rf voltage

$$
\overline{V_{\mathrm{c}}}=U_{\mathrm{dc}}+\left(U_{\mathrm{rf}} / k\right)
$$

drop across the 'cathode' sheath.

As follows from the formula (3.18) [19]

$$
J_{\mathrm{c}} / J_{\mathrm{a}}=A_{\mathrm{a}} / A_{\mathrm{c}},
$$

for the electrodes ('cathode' and 'anode') of equal area $\left(A_{\mathrm{c}}=\right.$ $A_{\mathrm{a}}$ ) the diffusion flows of positive ions out of the plasma to the boundaries of the sheaths are equal $J_{\mathrm{c}}=J_{\mathrm{a}}$. Then it is easy to obtain the relation between the thicknesses of the 'cathode' $d_{\mathrm{c}}$ and 'anode' $d_{\mathrm{a}}$ sheaths:

$$
d_{\mathrm{c}} / d_{\mathrm{a}}=\left[1+k \cdot\left(U_{\mathrm{dc}} / U_{\mathrm{rf}}\right)\right]^{3 / 5} .
$$

Figure 12 shows the ratios of thicknesses of the 'cathode' $d_{\mathrm{c}}$ and 'anode' $d_{\mathrm{a}}$ sheaths we registered for several values of the rf voltage applied. The range $x=U_{\mathrm{dc}} / U_{\mathrm{rf}} \leqslant 1.5$ corresponds to the first mode of the combined discharge and at $x>1.5$ the second mode is observed. The figure demonstrates that in the first mode the experimental data for $d_{\mathrm{c}} / d_{\mathrm{a}}$ are satisfactorily predicted by relation (8), the best fit corresponding to $k=\pi$. The calculation results according to formula (8) also agree with the registered data in the second mode.

The recent paper [31] reported the calculated expressions for the voltages across the sheaths and the sheath thicknesses in the longitudinal $\mathrm{rf} / \mathrm{dc}$ combined discharge. Using formulae 
(6), (8), (13), (19) and (20) from paper [31] we obtained the equation relating $d_{\mathrm{c}} / d_{\mathrm{a}}$ and $U_{\mathrm{dc}} / U_{\mathrm{rf}}$ :

$\frac{d_{\mathrm{c}}}{d_{\mathrm{a}}}=0.431$

$\times\left\{0.914+\left[\frac{5}{2 \xi} \frac{U_{\mathrm{dc}}}{U_{\mathrm{rf}}}\left(2+\chi\left(\frac{d_{\mathrm{c}}}{d_{\mathrm{a}}}-1\right)\right)+1.767\right]^{3 / 5}\right\}$,

where for the collisional case $\chi=1.52, \xi=0.78$. It is clear from figure 12 that the calculation data according to equation (9) agree with the registered data at $U_{\mathrm{dc}} / U_{\mathrm{rf}}<1$, whereas at higher $U_{\mathrm{dc}} / U_{\mathrm{rf}}$ values equation (9) furnishes higher $d_{\mathrm{c}} / d_{\mathrm{a}}$ values and at $U_{\mathrm{dc}} / U_{\mathrm{rf}}>2.6$ equation (9) loses the physical sense because it predicts negative $d_{\mathrm{c}} / d_{\mathrm{a}}$ values (it is not shown in figure 12).

\section{Conclusion}

We studied the longitudinal combined discharge ( $\mathrm{rf}+\mathrm{dc}$ voltages were applied to the same electrodes) in low pressure nitrogen. Three modes of such a discharge are shown to exist. In the first mode (non-self-sustained rf discharge perturbed with the dc electric field), ionization takes place in the plasma volume in the rf field and the conductance of the 'cathode' as well as 'anode' sheaths is small. In the second mode (the combined discharge) the 'cathode' sheath is broken by the joint action of the dc and rf electric fields, and electron avalanches develop in it. This mode is of considerable interest for plasma technologies. The third mode (non-self-sustained $\mathrm{dc}$ discharge perturbed with the rf field) is observed when a weak rf voltage is applied to the dc discharge. It is shown that the region where only the first mode exists is limited from the low pressure side by the rf discharge extinction curve as well as the transition curve of the combined discharge separating the ranges of the first mode and the second one. In this range of the rf voltage and gas pressure the increasing dc voltage may extinguish the combined discharge burning in the first mode. From the Child-Langmuir law for the collisional rf sheath a relation is obtained between the thicknesses of the 'cathode' and 'anode' near-electrode sheaths for the first mode which furnishes a good description of the experimental data.

\section{References}

[1] Varnerin L J and Brown S C 1950 Phys. Rev. 79946

[2] Lisovskii V A 1998 Tech. Phys. Lett. 24308

[3] Cristescu G D 1960 Ann. Phys. 7153

[4] Ligenza J R 1965 J. Appl. Phys. 362703

[5] Batinin V M, Roddatis V K 1981 High Temp. 18844

[6] Eckbreth A C and Davis J W 1972 Appl. Phys. Lett. 2125

[7] Brown C O and Davis J W 1972 Appl. Phys. Lett. 21480

[8] Yamamoto K and Okuda T 1956 Appl. Sci. Res. 5144

[9] Goichman V H, Goldfarb V M and Tendler M B 1975 Proc. 12th Int. Conf. on Phenomena in Ionized Gases (Eindhoven, The Netherlands) p 109

[10] Rakhimova T V and Rakhimov A T 1975 Sov. J. Plasma Phys. 1468

[11] Myshenkov V I and Yatsenko N A 1982 Sov. J. Plasma Phys. 8397

[12] Lisovskii V A and Yegorenkov V D 1992 Soviet Tech.—Phys. Lett. 18573

[13] Lisovskii V A 1992 Soviet Techn. Phys. Lett. 18616

[14] Lisovsky V A and Yegorenkov V D 1994 J. Phys. D: Appl. Phys. 272340

[15] Zeuner M and Meichsner J 1995 Surf. Coat. Technol. 74-75 562

[16] Hassan Z and Fujita H 1998 J. Phys. D: Appl. Phys. 312281

[17] Shoji M and Sato M 1999 J. Phys. D: Appl. Phys. 321640

[18] Radmilovic-Radjenovic M and Radjenovic B 2006 Plasma Sources Sci. Technol. 151

[19] Raizer Y P, Shneider M N and Yatsenko N A 1995 Radio-Frequency Capacitive Discharges (Boca Raton, FL: CRC Press)

[20] Raizer Y P 1991 Gas Discharge Physics (Berlin: Springer)

[21] Zakrzewski Z and Kopiczynski T 1974 Plasma Phys. 161195

[22] Tichy M, Sicha M, David P and David T 1994 Contrib. Plasma Phys. $\mathbf{3 4} 59$

[23] Lisovskiy V, Booth J-P, Landry K, Douai D, Cassagne V and Yegorenkov V 2006 Phys. Plasmas 13103505

[24] Lisovskiy V, Booth J-P, Martins S, Landry K, Douai D and Cassagne V 2005 Europhys. Lett. 71407

[25] Guerra V, Sa P A and Loureiro J 2004 Eur. Phys. J. Appl. Phys. 28125

[26] Belenguer Ph and Boeuf J P 1990 Phys. Rev. A 414447

[27] Godyak V A and Kuzovnikov A A 1975 Sov. J. Plasma Phys. 1276

[28] Lieberman M A and Lichtenberg A J 1994 Principles of Plasma Discharges and Materials Processing (New York: Wiley)

[29] Razer Yu P and Shneder M N 1994 Tech. Phys. Lett. 20806

[30] Lieberman M A 1989 IEEE Trans. Plasma Sci. 17338

[31] Kawamura E, Lieberman M A, Lichtenberg A J and Hudson E A 2007 J. Vac. Sci. Technol. A 251456 